\title{
Knockdown of MSI1 inhibited the cell proliferation of human osteosarcoma cells by targeting p21 and p27
}

\author{
JIANBING NIU $^{1}$, XIULIAN ZHAO ${ }^{2}$, QINGSHENG LIU ${ }^{1}$ and JINSAN YANG ${ }^{1}$ \\ ${ }^{1}$ Department of Bone and Joint Surgery, Shandong Jining No. 1 People's Hospital, Jining, Shandong 272011; ${ }^{2}$ Department of \\ Kidney and Chinese Medicine, Shandong Jinxiang County People's Hospital, Jinxiang, Shandong 272200, P.R. China
}

Received October 24, 2016; Accepted July 13, 2017

DOI: $10.3892 / \mathrm{ol} .2017 .6870$

\begin{abstract}
Osteosarcoma is the most common type of primary bone cancer in children and adolescents, but its mechanism remains unclear. Musashi RNA-binding protein 1 (MSI1) is highly expressed in certain cancer types and functions as a putative progenitor/stem cell marker. In the present study, it was demonstrated that MSI1 expression in osteosarcoma tissue was higher compared with in the paraneoplastic tissue samples. Knockdown of MSI1 using shRNA in MG-63 and HOS cells inhibited cell proliferation in vitro and tumor formation in vivo, suggesting that MSI1 serves an essential role in osteosarcomagenesis. Further investigations demonstrated that the knockdown of MSI1 leads to the cell cycle arrest at $\mathrm{G}_{0} / \mathrm{G}_{1}$ phase, and the upregulation of p21 and p27 protein expression in osteosarcoma cells. Additionally, luciferase assays demonstrated that MSI1 can bind to the 3' untranslated regions of p21 and p27 mRNA. In conclusion, the results of the present study suggest that the knockdown of MSI11 can suppress cell proliferation of osteosarcoma by targeting p21 and p27 and subsequently inhibiting cell cycle progression.
\end{abstract}

\section{Introduction}

Osteosarcoma is the most common non-haematological primary malignant bone tumor that occurs in children and adolescents and its overall relapse-free survival rate over 5 years is $65-75 \%$ (1). Osteosarcoma is characterized by a highly malignant and metastatic potential, and the leading cause of death of osteosarcoma patients is distant metastases (2). However, at present, the pathogenesis of osteosarcoma remains unclear.

Increasing evidences have showed that tumor stem cells are considered to be responsible for the metastasis of

Correspondence to: Professor Jinsan Yang, Department of Bone and Joint Surgery, Shandong Jining No. 1 People's Hospital, 6 Jiankang Road, Jining, Shandong 272011, P.R. China

E-mail: yangjinshan559@163.com

Key words: Musashi RNA-binding protein 1, osteosarcoma, cell proliferation, p21, p27 tumors (3), and some stem cell-related genes are involved in tumorgenesis $(4,5)$. MSI1 is a RNA-binding protein of the Musashi family involved in early asymmetric divisions generating differentiated cells from neural stem cells or progenitor cells. MSI1 is highly enriched in the nervous system and has been found to be related with the grade of the malignancy in glioma (6). Additionally, MSI1 drives progenitor cell expansion along the luminal and myoepithelial lineages in mammary glands and regulates the proliferation and apoptosis of mesenchymal stem cells. Recently, high MSI1 expression has been found in various types tumors, including medulloblastoma (7), colon cancer (8), lung cancer (9), cervical cancer (10) and breast cancer (11), and appeared to be a maker of poor prognosis. Moreover, MSI1 has been found to activate the Notch and Wnt signaling pathways in several types of normal and cancerous cells $(12,13)$. However, the role of MSI1 in osteosarcoma progression is currently unclear.

Here, we investigated the function of MSI1 and its mechanism in the progression of osteosarcoma. In this study, we found that MSI1 expression is increased in osteosarcoma tissue compared with paraneoplastic tissue. Knockdown of MSI1 resulted in the decreased cell proliferation and slow growth of the tumor xenografts. Furthermore, knockdown of MSI1 resulted in the arrest of cell cycle and up-regulation of p21 and p27 protein expression. These results supported that MSI1 functions as an oncogene in osteosarcoma.

\section{Materials and methods}

Tissue samples. A total of 30 Fresh frozen specimens of matched osteosarcoma tissues and paraneoplastic tissues were collected from Shandong Jining No. 1 People's Hospital (Jining, China) from 2011 to 2014. None of the patients had received chemotherapy, immunotherapy or radiotherapy before the specimen collection. The study was approved by Institutional Research Ethics Committee, and patients gave their informed consent before sample collection.

Cell lines and cell culture. Human osteosarcoma cell lines, MG-63 and HOS, were purchased from the American Type Culture Collection (Rockville, MD, USA) and were cultured in Dulbecco's modified Eagle's medium (DMEM; Sigma-Aldrich, St. Louis, MO, USA) supplemented with $10 \%$ fetal bovine 
serum (FBS; Invitrogen; Thermo Fisher Scientific, Inc., Waltham, MA, USA) and $1 \%$ penicillin-streptomycin. All cells were cultured at $37^{\circ} \mathrm{C}$ in an atmosphere $5 \% \mathrm{CO}_{2}$.

Vector construction. The small interfering RNA expression vector that expresses MSI1-specific short hairpin RNA (shRNA) was purchased from GenePharma Co., Ltd. (Shanghai, China). To construct reporter vector containing the 3'UTR of p21 and p27, the fragments of the 3'UTR of p21 and p27 mRNA were separately extracted from MG-63 cells and amplified from cDNA by PCR using primers listed below, then cloned into the pMIR-REPORT luciferase vector. The following primers were listed in Table I.

Western blot analysis. Cells and clinical tissues were lysed on ice in lysis buffer containing freshly added protease inhibitor cocktail (Roche Diagnostics, Branchburg, NJ, USA). The protein extracts $(15 \mu \mathrm{g})$ were separated using SDS-PAGE and transferred into polyvinylidene difluoride (PVDF) membranes (EMD Millipore, Billerica, MA, USA). The appropriate primary antibodies were used after the membranes were blocked in $5 \%$ fat-free milk. The primary antibodies included the following: anti-MSI1 $(1: 1,000$, cat. no. sc-98845; Santa Cruz Biotechnology, Inc., Santa Cruz, CA, USA), anti-p21 (1:500, cat. no. sc-397; Santa Cruz Biotechnology, Inc.), anti-p27 (1:500, cat. no. sc-397; Santa Cruz Biotechnology, Inc.) and anti- $\beta$-actin (1:500, cat. no. sc-47778; Santa Cruz Biotechnology, Inc.). Blots were incubated with a secondary antibody coupled to horseradish peroxidase (Thermo Fisher Scientific Inc.), and visualized on X-ray film. Relative quantitation was measured using the AlphaView system (Cell Biosciences, Santa Clara, CA, USA).

RT-qPCR analysis. Total RNA was extracted from cells with TRIzol Reagent (Invitrogen; Thermo Fisher Scientific, Inc.) and reverse transcription reactions were performed using RT-PCR kit (Takara Biotechnology Co., Ltd., Dalian, China). Relative mRNA levels were evaluated by quantitative PCR using SYBR-Green Mastermix (Takara Biotechnology Co., Ltd.). The primer sequences were listed in Table II. The results were analyzed according to the $\mathrm{Cq}(\Delta \Delta \mathrm{Cq})$ method using GAPDH as the normalizing gene.

MTT assays. Cell viability was assessed every other day using 3-(4,5-dimethylthiazole-yl) 2,5-diphenyl tetrazolium bromide (MTT, Sigma-Aldrich, USA) dye according to standard protocol. Approximately $2 \times 10^{3}$ cells/well were seeded in a 96-well plate and incubated for 7 days. $20 \mu \mathrm{l}$ of MTT solution was added to $200 \mu \mathrm{l}$ of culture media and incubated for $4 \mathrm{~h}$, and then dissolved in $100 \mu \mathrm{l}$ of dimethylsulphoxide (DMSO; Sigma-Aldrich). Cell proliferation was determined by measuring the absorbance at $490 \mathrm{~nm}$.

Flow cytometry analysis. Cells $\left(2 \times 10^{6}\right)$ were harvested and fixed with $70 \%$ cold ethanol at $4{ }^{\circ} \mathrm{C}$ overnight. After washed twice in PBS, the cells were suspended in PBS with $50 \mu \mathrm{g} / \mathrm{ml}$ propidium iodide and $10 \mu \mathrm{g} / \mathrm{ml} \mathrm{RNaseA}$, and then incubated at room temperature for $30 \mathrm{~min}$ in the dark. Then, the cells were measured by FACSCalibur flow cytometry (BD Biosciences,
Franklin Lakes, NJ, USA), and the cell cycle distributions were analyzed.

In addition, the cells were harvested, washed in PBS and stained in duplicate with APC annexin V and propidium iodide (BD Pharmingen, Franklin Lakes, NJ, USA) for $10 \mathrm{~min}$ in the dark to characterize cell apoptosis using flow cytometry.

Animal and tumor xenograft assay. Tumor cells collected from stable transfectants were bilaterally injected into subcutis on the dorsum of 6- to 7-week-old Balb/c nude mice. Tumor size was measured with calipers once every week, and volumes $\left(\mathrm{cm}^{3}\right)$ were calculated according to the standard formula: $V=$ length $\mathrm{x}$ width ${ }^{2} / 2$. At the end of the experiment, tumors were dissected out, and the net weight was measured. All animals received humane treatment in accordance with institutional policies, and all experimental protocols were approved by the Animal Care and Use Committee of Jining First people's Hospital.

Luciferase reporter assay. In brief, plasmids containing firefly luciferase reporters were cotransfected into cells using Lipofectamine ${ }^{\circledR} 2000$ (Invitrogen; Thermo Fisher Scientific, Inc.). Then, the cells were lysed in $100 \mathrm{ul}$ of passive lysis buffer (Promega Corporation, Madison, WI, USA) at $48 \mathrm{~h}$ after transfection and determined with a dual-luciferase assay according to the manufacturer's instructions. Luciferase activities were detected in a luminometer (Promega Corporation) and expressed at the ratio of Firefly to Renilla luciferase activity.

Statistical analysis. All results were confirmed in three independent experiments, and data were expressed as the mean \pm standard deviation. Student's t-test or one-way ANOVA test were performed using SPSS 16.0 (SPSS, Inc., Chicago, IL, USA). $\mathrm{P}<0.05$ was considered to indicate a statistically significant difference.

\section{Results}

The expression of MSII was upregulated in osteosarcoma. To determine the expression of MSI1 in osteosarcoma, Western blot analysis was conducted. We found the expression of MSI1 in osteosarcoma tissues was markedly higher than that in the paraneoplastic tissues $(\mathrm{P}<0.01$, Fig. 1A). Additionally, the expression of MSI1 in osteosarcoma cell lines was also measured by western blot, and the high expression of MSI1 in U-2OS, HOS, Hs-188T and MG-63 cell lines were observed (Fig. 1B). These data suggested MSI1 may contribute to the progression of osteosarcoma.

The effect of knockdown of MSII on the proliferation and apoptosis of osteosarcoma cells. To assess the effect of MSI1 on the proliferation and apoptosis of osteosarcoma cells, we established MG-63 and HOS osteosarcoma cells with MSI1 knockdown by shRNA, respectively (Fig. 2A and B). MTT assay revealed that MSI1 knockdown MG-63 cells had much lower proliferation ability than the corresponding control cells (Fig. 2C), and similar results were observed in MSI1 knockdown HOS cells (Fig. 2D). These findings suggested that 
Table I. The primer sequence of p21 and p27.

\begin{tabular}{lll}
\hline Gene & Orientation & Primer sequence \\
\hline WT p21 & Forward & 5'-ATTGAGCTCTAATCCGCCCACAGGAAG-3' \\
& Reverse & 5'-CTCAAGCTTACAAGTAAAGTCACTAAG-3' \\
MU p21 & Forward & 5'-TGGGAACGACTGTCTTTCCTGGCACTAACGTT-3' \\
& Reverse & 5'-AGACAGTCGTTCCCAGCCCCATATGAGCCCAC-3' \\
WT p27 & Forward & F' 5'-CGCGAGCTCGAATTAAGAATATGTTTC-3' \\
MU p27 & Reverse & 5'-TTGACGCGTATGCAACCTTTTAAGCATAGC-3' \\
& Forward & 5'-CCGCTCGAGTGATCTGCCTCTAAAAGCGT-3' \\
& Reverse & 5'-CGGGATCCATTCTTAACATTCAAAACTCCC-3' \\
\hline
\end{tabular}

WT, wild-type; MU, mutant.

Table II. The primer sequence of p21 and p27.

\begin{tabular}{llc}
\hline Genes & Orientation & Primer sequence \\
\hline p21 & Forward & 5'-GAGCAGTGCCCGAGTTAAGG-3' \\
& Reverse & 5'-TGGAACAGGTCGGACATCAC-3' \\
p27 & Forward & 5'-GGTGCCTTCAATTGGGTCTC-3' \\
& Reverse & 5'-GCTTCCTCATCCCTGGACAC-3' \\
\hline
\end{tabular}

knockdown of MSI1 suppressed the proliferation of osteosarcoma cells.

However, the apoptosis analysis of MG-63 and HOS cells using flow cytometry showed that there was no significant difference in the proportion of apoptotic cells between MSI1 knockdown cells and the control (Fig. 2E and F), which suggested that MSI1 had no significant influence on apoptosis in the osteosarcoma cells.

Knockdown of MSII inhibited the tumor formation of osteosarcoma cells. To further assess the effect of MSI1 on the tumor formation, nude mice were injected subcutaneously with MSI1 knockdown MG-63 and HOS cells, and the growth of tumors was monitored in terms of tumor volume every three days. At the end of the experiment, the mice were sacrificed, and the tumors were excised and weighed. The tumors formed by the MSI1 knockdown MG-63 and HOS cells grew much slower than those formed by the Control (Fig. 3A and B, $\mathrm{P}<0.01)$. In addition, the weights of the tumors formed by the MSI1 knockdown cells were significantly reduced compared to the Control (Fig. 3C and D, P<0.01). All these data suggested that knockdown of MSI1 inhibited the tumor formation ability of osteosarcoma cells in vivo.

Knockdown of MSII led to the cell cycle arrest of osteosarcoma cells. We all know that cell proliferation is usually associated with the modulation of the cell cycle, then, we characterized cell cycles by flow cytometry analysis. As shown in Fig. 4A and B, the proportion of MSI1 knockdown MG-63 cells in G0/G1 phase was markedly increased, while proportion in $\mathrm{S}$ phase was significantly decreased compared to the control $(\mathrm{P}<0.05)$, and a similar result was observed in MSI1 knockdown HOS cells (Fig. 4C and D). These data indicated that the knockdown of MSI1 resulted in the cell cycle arrest.

Knockdown of MSII increased the expression of p21 and p27 protein. Previous research reported that MSI1 regulated cell cycle by negative regulation of p21 expression in bladder carcinoma (14) and breast cancer (15). To explore the molecular mechanism of MSI1 on the regulation of cell cycle of osteosarcoma cells, the expression of p21 and p27 were examined by Western blot (Fig. 5A-D). A significant increase in the levels of p21 and p27 protein expression were observed in the MSI1 knockdown MG-63 and HOS cells compared to their control cells, respectively $(\mathrm{P}<0.01)$, suggesting that MSI1 may reduce the levels of p21 and p27 protein expression. However, the examination using RT-qPCR assay showed that no significant differences in the expression of p21 and p27 mRNA were observed in the MSI1 knockdown MG-63 and HOS cells and their control (Fig. 5E and F). Therefore, these data indicated that MSI1 could influence the expression of p21 and p27 at protein level, not mRNA level in MG-63 and HOS cells.

It has been reported that p21 and p27 were the MSI1 target genes in which there is a binding sequence region in their 3'UTR $(16,17)$. The MSI1 binding sites in p21 and p27 protein are shown in Fig. 6A, respectively. To test the direct interaction between MSI1 and its putative target site, the wild-type and mutant MSI1 binding sequence was inserted downstream of the luciferase vectors (Fig. 6B). After transfected by p21 wild-type vector, the relative luciferase activity of MSI1 knockdown MG-63 and HOS cells was significantly increased compared to the control $(\mathrm{P}<0.01)$, however, no significant difference in the luciferase activities between the MSI1 knockdown MG-63 and HOS cells and their control cells transfected with the mutant vector was observed (Fig. 6C and D). Similarly, the luciferase activity levels in MSI1 knockdown MG-63 and HOS cells were found to be higher than their control cells after being transfected with the p27 1\&2 wild-type vector $(\mathrm{P}<0.01)$, while, the differences were not significant between the MSI1 knockdown MG-63 and HOS cells and their control cells after being transfected by p27 $1 \& 2$ mutant vector. These data suggested that MSI1 


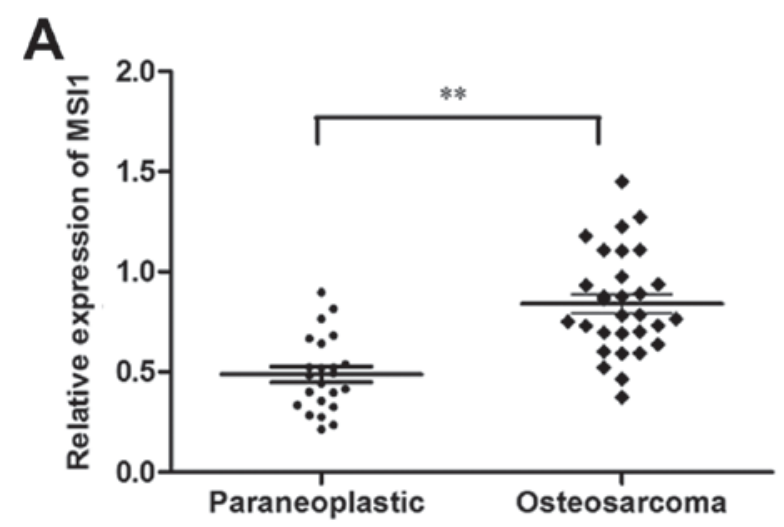

B

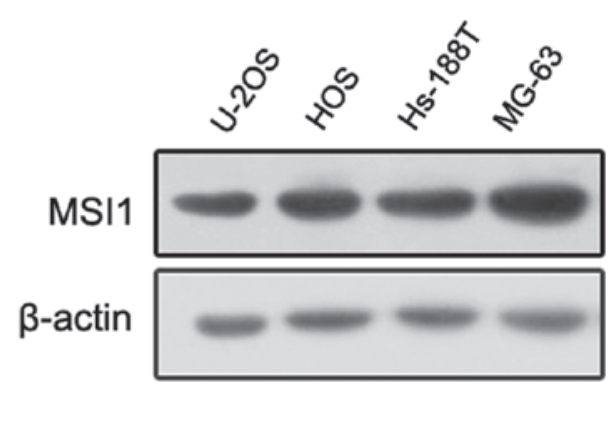

Figure 1. The expression of Musashi RNA-binding protein 1 (MSI1) in osteosarcoma tissues, paraneoplastic tissues and osteosarcoma cell lines. (A) MSI1 protein expression in osteosarcoma tissues was higher than that of the paraneoplastic tissues ( ${ }^{* *} \mathrm{P}<0.01$ ). (B) MSI1 expression in human osteosarcoma cell lines was shown by western blot analysis.

A

MG-63

Con shCon shMSI1

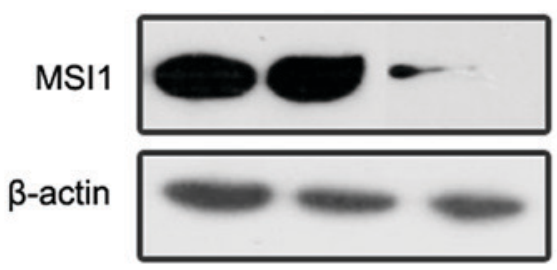

C

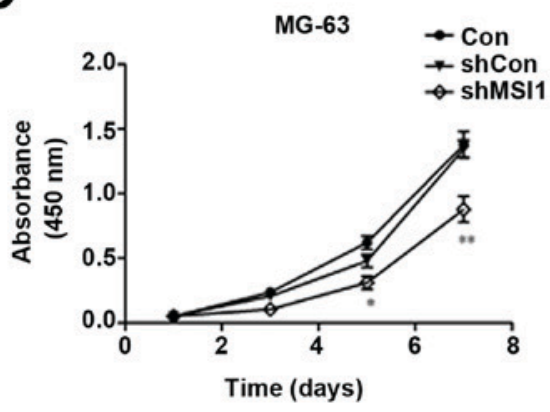

E

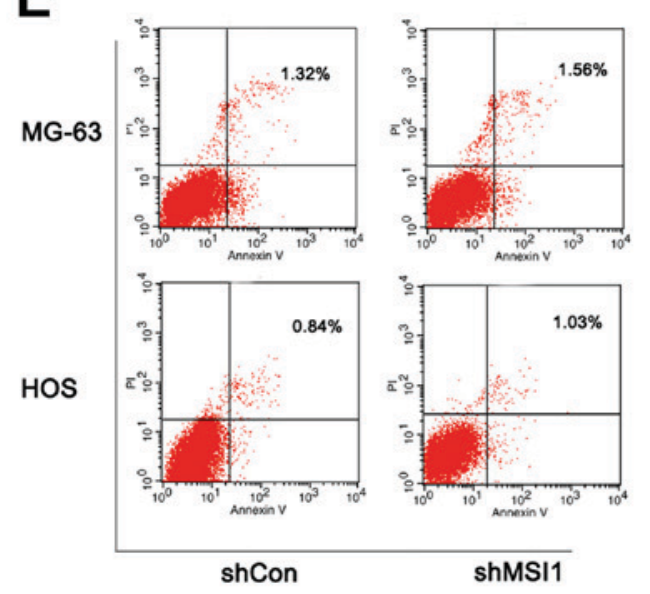

B

HOS

Con shCon shMSI1

MSI1

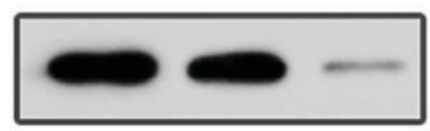

$\beta$-actin

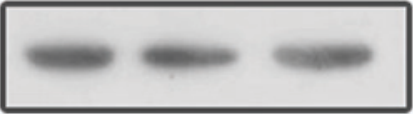

D

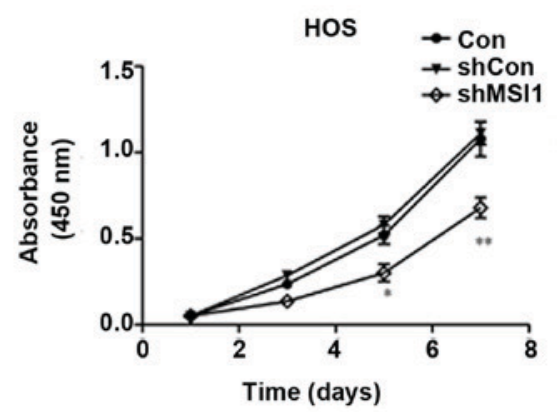

F

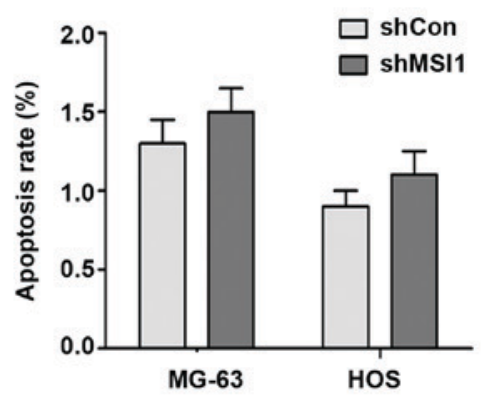

Figure 2. The effect of knockdown of Musashi RNA-binding protein 1 (MSI1) on the proliferation and apoptosis of osteosarcoma cells. Western blot analysis of the expression of MSI1 in (A) MG-63 and (B) HOS cells with MSI1 knockdown by shRNA. The cell proliferation of MSI1 knockdown (C) MG-63 and (D) HOS cells were evaluated by MTT assay. (E) Apoptosis assay for MG-63 and HOS was performed and (F) quantitative analysis. * P $<0.01$ compared to the shCon. 
A

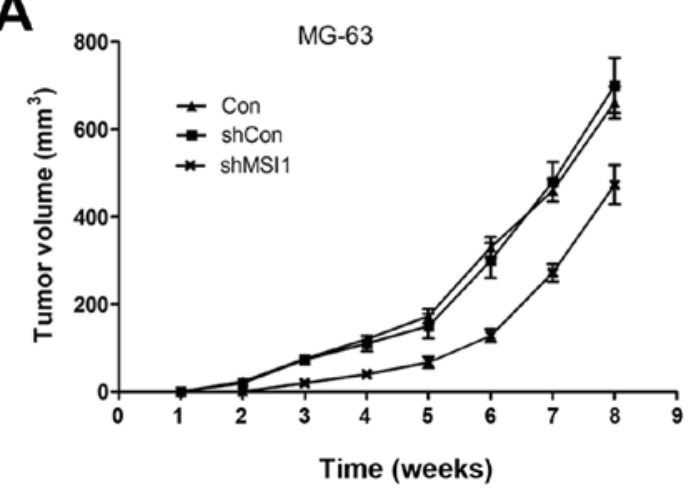

C

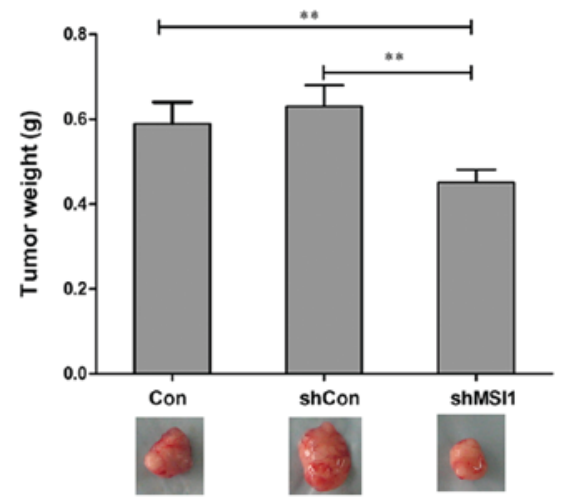

B

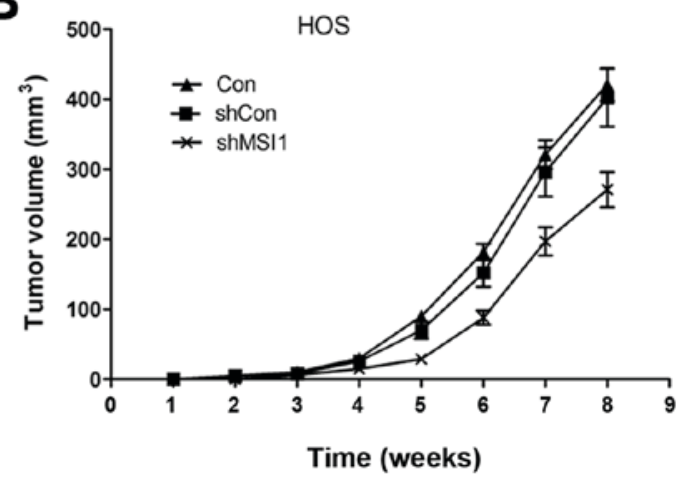

D

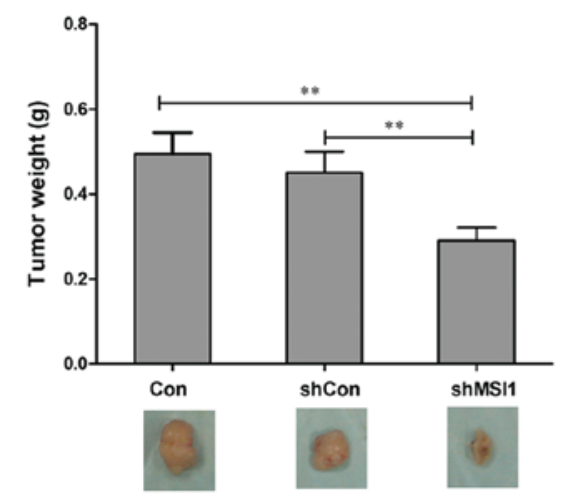

Figure 3. Knockdown of Musashi RNA-binding protein 1 (MSI1) inhibited the growth of xenograft tumors in vivo. The growth of xenograft tumors formed by MSI1 knockdown (A) MG-63 and (B) HOS cells were monitored longitudinally for 8 weeks. (C and D) At end of the experiment, the tumors were dissected out, and the tumor wet weights were measured. ${ }^{* *} \mathrm{P}<0.01$ compared to the shCon.

A

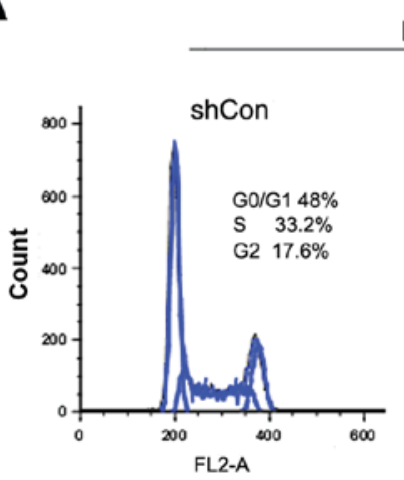

MG-63

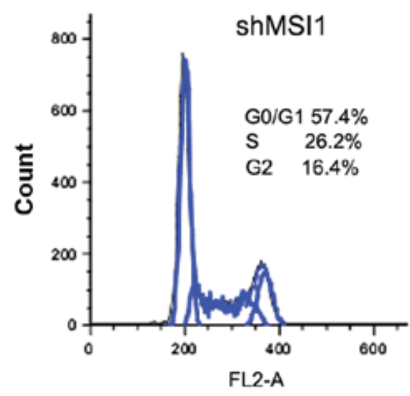

C

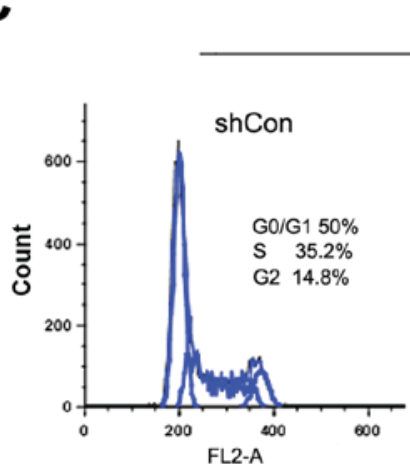

HOS

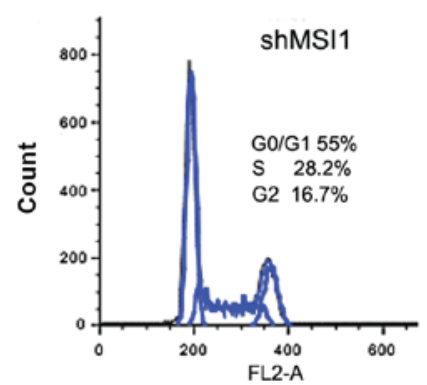

B

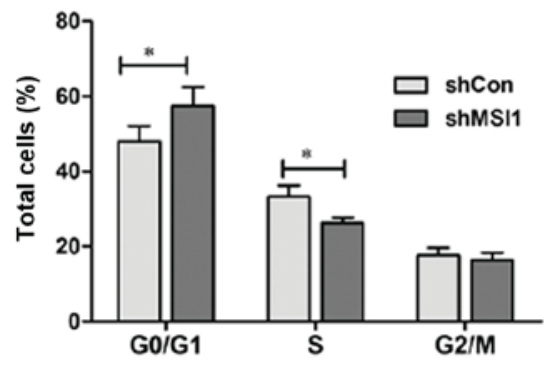

D

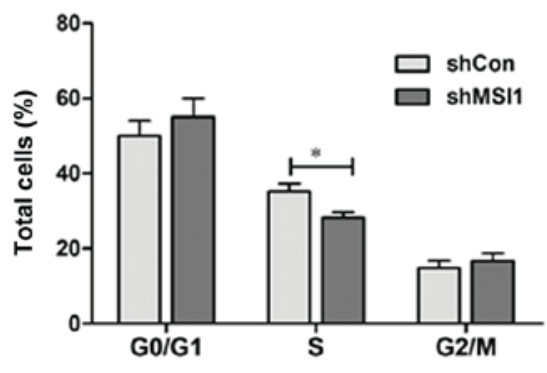

Figure 4. The effect of Knockdown of Musashi RNA-binding protein 1 (MSI1) on cell cycle and apoptosis of MG-63 and HOS cells. Cell cycle analysis was performed by flow cytometry, and the representative cell cycle histograms of MSI1 knockdown (A) MG-63 and (C) HOS cells were shown. Quantitative analysis was shown for the cell cycle distribution of (B) MG-63 and (D) HOS. " $\mathrm{P}<0.05$ compared to the shCon. 
A

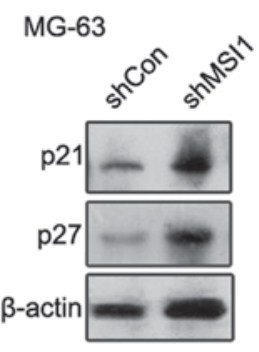

C

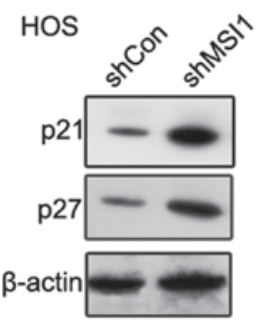

E

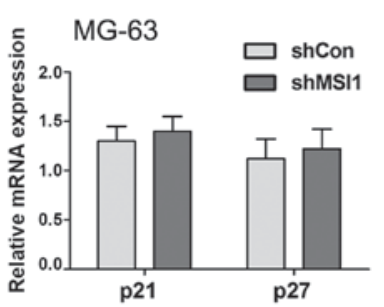

B

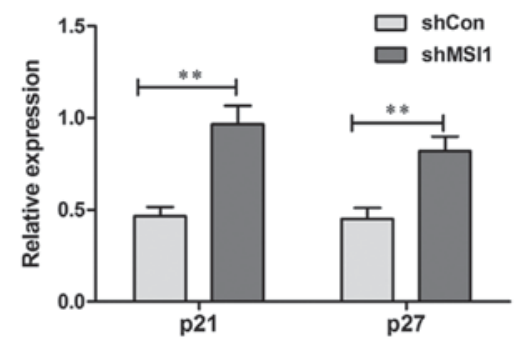

D

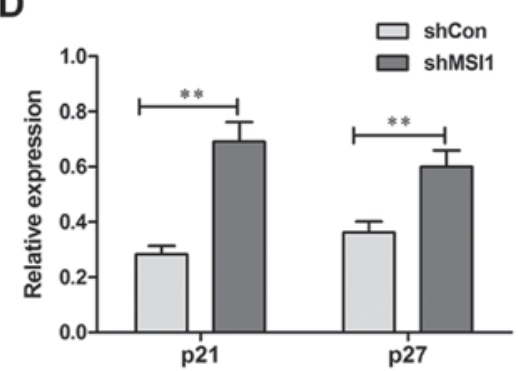

$\mathbf{F}$

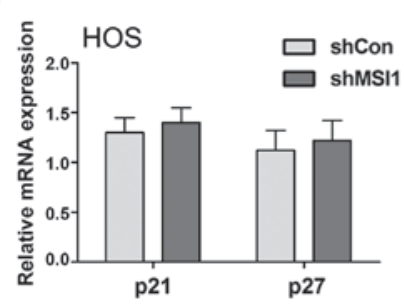

Figure 5. Musashi RNA-binding protein 1 (MSI1) regulated the expression of $\mathrm{p} 21$ and $\mathrm{p} 27$ at protein level. The expression of p21 and p27 proteins in MSI1 knockdown (A) MG-63 and (C) HOS cells were measured by western blot assay, and (B and D) the relative expression of p21 and p27 were calculated based on $\beta$-actin. The levels of p21 and p27 transcripts in MSI1 knockdown (E) MG-63 and (F) HOS cells were measured by qPCR. ** P $<0.01$ compared to the shCon.

A

WT P21 3'UTR 5'-TGGGGCTGGGAGTAGTTGTCTTTCCTGGCACT-3' MU P21 3'UTR 5'-T GGGGCTGGGAACGACTGTCTTTCCTGGCACT-3'

WT1\&WT2 P27 3'UTR

5-TGtatatagatTtTACCTtTTATGTAGCA-3' 5'-CCAGGTAGTTTGGGGCAAAAATCCGAG-3'

MU1\& MU2 P27 3'UTR

5'-TGTATGGCAGTTTTACCTITTATGTAGCA-3'

5'-CCAGAGCAGTTGGGGCAAAAATCCGAG-3'

C

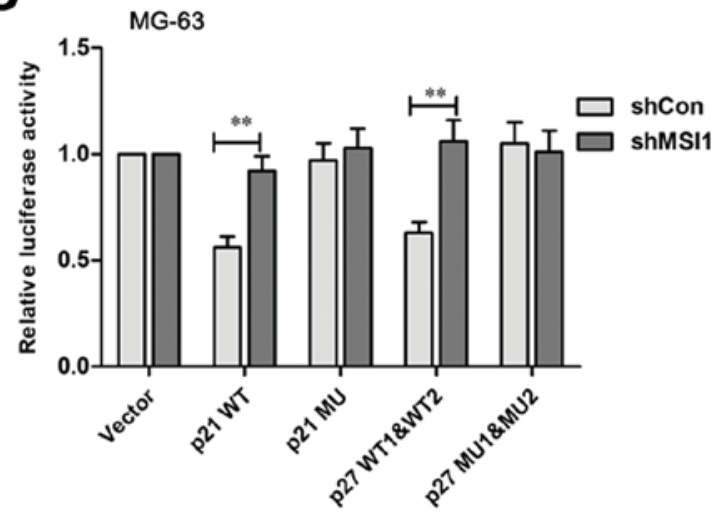

B

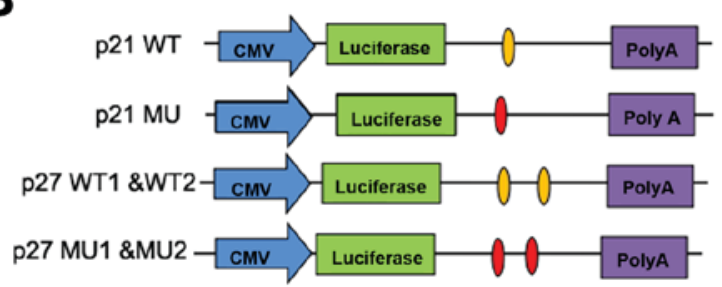

D

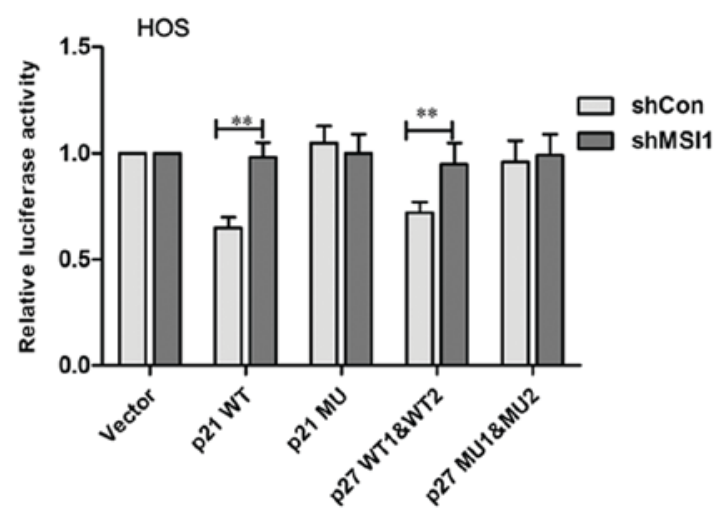

Figure 6. Musashi RNA-binding protein 1 (MSI1) binded to the 3'UTR of p21 and p27. (A) Schematic construction of MSI1 binding sites with wild-type (WT) and mutant (MU) 3'UTR of p21 and p27 as predicted. (B) Schematic representation of the reporter construct containing the firefly luciferase coding sequence fused to p21 and p27 3'UTR. The predicted binding site regions within the wild-type p21 and p27 3'UTR are noted in orange ovals, and the residues altered in the p21 3'UTR/p27 3'UTR mutants construct are marked on the red ovals. Luciferase reporter assays for p21 and p27 targeted by MSI1 in (C) MG-3 and (D) HOS cells, and the histogram shows the ratio of firefly to Renilla luciferase activity normalized to empty vector transfected cells. ${ }^{* *} \mathrm{P}<0.01$ compared to the shCon. 
might inhibit p21 and p27 translation by specifically binding site.

\section{Discussion}

Increasing evidence showed that RNA binding proteins play an important role in the cell proliferation and apoptosis involved in tumorigenesis (18-20). MSI1, a multifunction RNA binding protein of MSI family, was initially identified as a marker of neuronal stem cell $(21,22)$ and more recently identified as a putative marker of intestinal stem cell (23), was overexpressed in several types of cancers, such as glioma (13), medulloblastoma (24), and breast cancer (15).

In this study, we found that the expression of MSI1 protein in osteosarcoma tissues was significantly higher than that of the paraneoplastic tissues, suggesting MSI1 was likely to play an important role in osteosarcomagenesis. To further seek for the function of MSI1 involved in osteosarcomagenesis, the functional characterization was conducted by knockdown of MSI1 in osteosarcoma cell lines (MG-63 and HOS cells). We found that knockdown of MSI1 significantly inhibited the proliferation of MG-63 and HOS cells in vitro. Moreover, knockdown of MSI1 also significantly suppressed the tumor growth in vivo. These results suggested that MSI1 played an important role in the cell proliferation and tumor growth. In addition, the analysis of cell cycle and apoptosis showed that knockdown of MSI1 induced cell cycle arrest in the G0/G1 phase, but no markedly change of apoptosis, suggesting that MSI1 might have important impact on the cell cycle, not apoptosis. It is well known that cell proliferation is usually regulated by cell cycle progression. Therefore, our data suggested that the inhibitory effect of MSI1 knockdown on cell proliferation and tumor formation of osteosarcoma cells were probably associated with the regulation of cell cycle. Our finding was in agreement with previous findings in other types of tumors (25-27), suggesting that MSI1 related signaling may have a similar regulatory effect on the growth of different types of human malignancies.

It has been reported that increased expression of p21 and p27 leads to apoptosis and cell cycle arrest in various cancer cells $(16,28,29)$. In this study, we found the expression of p21 and p27 protein levels were up-regulated in the MSI1 knockdown MG-63 and HOS cells compared to the control. As p21 and p27 is universal cyclin-dependent kinase (CDK) inhibitor that directly inhibits the activity of cyclin-CDK complexes $(30,31)$, resulting in cell cycle arrest at $\mathrm{G} 0 / \mathrm{G} 1$ phase, these results suggested that the cell cycle arrest caused by MSI1 knockdown in osteosacoma cells might be associated with the overexpression of p21 and p27. Furthermore, luciferase assay revealed that MSI could directly bind to the consensus sequence of p21 and p27 3'UTR in osteosarcoma cells, indicating that knockdown of MSI1 could lead to the up-regulation of the expression of p21 and p27.

In summary, our study demonstrated that MSI1 was high expressed in osteosarcoma tissue, and knockdown of MSI1 inhibited the cell proliferation and tumor formation by the arrest of cell cycle involved the activation of p21 and p27. Our study provided a potential target for clinical therapy of osteosarcoma.

\section{References}

1. Lewis IJ, Nooij MA, Whelan J, Sydes MR, Grimer R, Hogendoorn PC, Memon MA, Weeden S, Uscinska BM, van Glabbeke M, et al: Improvement in histologic response but not survival in osteosarcoma patients treated with intensified chemotherapy: A randomized phase III trial of the European osteosarcoma intergroup. J Natl Cancer Inst 99: 112-128, 2007.

2. Buddingh EP, Anninga JK, Versteegh MI, Taminiau AH Egeler RM, van Rijswijk CS, Hogendoorn PC, Lankester AC and Gelderblom H: Prognostic factors in pulmonary metastasized high-grade osteosarcoma. Pediatr Blood Cancer 54: 216-221, 2010.

3. Kapoor A and Kumar S: Cancer stem cell: A rogue responsible for tumor development and metastasis. Indian J Cancer 51: 282-289, 2014.

4. Liu XF, Yang WT, Xu R, Liu JT and Zheng PS: Cervical cancer cells with positive Sox 2 expression exhibit the properties of cancer stem cells. PLoS One 9: e87092, 2014.

5. Wu XS, Xi HQ and Chen L: Lgr5 is a potential marker of colorectal carcinoma stem cells that correlates with patient survival. World J Surg Oncol 10: 244, 2012

6. Toda M, Iizuka Y, Yu W, Imai T, Ikeda E, Yoshida K, Kawase T, Kawakami Y, Okano H and Uyemura K: Expression of the neural RNA-binding protein Musashil in human gliomas. Glia 34: 1-7, 2001.

7. Sanchez-Diaz PC, Burton TL, Burns SC, Hung JY and Penalva LO: Musashil modulates cell proliferation genes in the medulloblastoma cell line Daoy. BMC Cancer 8: 280, 2008.

8. Fan LF, Dong WG, Jiang CQ, Xia D, Liao F and Yu QF: Expression of putative stem cell genes Musashi-1 and beta1-integrin in human colorectal adenomas and adenocarcinomas. Int J Colorectal Dis 25: 17-23, 2010.

9. Wang XY, Yu H, Linnoila RI, Li L, Li D, Mo B, Okano H, Penalva LO and Glazer RI: Musashil as a potential therapeutic target and diagnostic marker for lung cancer. Oncotarget 4: 739-750, 2013.

10. Ye F, Zhou C, Cheng Q, Shen J and Chen H: Stem-cell-abundant proteins Nanog, Nucleostemin and Musashil are highly expressed in malignant cervical epithelial cells. BMC Cancer 8: 108, 2008.

11. Wang XY, Penalva LO, Yuan H, Linnoila RI, Lu J, Okano H and Glazer RI: Musashi1 regulates breast tumor cell proliferation and is a prognostic indicator of poor survival. Mol Cancer 9: 221, 2010.

12. Wang XY, Yin Y, Yuan H, Sakamaki T, Okano H and Glazer RI: Musashil modulates mammary progenitor cell expansion through proliferin-mediated activation of the Wnt and Notch pathways. Mol Cell Biol 28: 3589-3599, 2008.

13. Muto J, Imai T, Ogawa D, Nishimoto Y, Okada Y, Mabuchi Y, Kawase T, Iwanami A, Mischel PS, Saya H, et al: RNA-binding protein Musashi1 modulates glioma cell growth through the post-transcriptional regulation of Notch and PI3 kinase/Akt signaling pathways. PLoS One 7: e33431, 2012.

14. Nikpour P, Baygi ME, Steinhoff C, Hader C, Luca AC, Mowla SJ and Schulz WA: The RNA binding protein Musashi1 regulates apoptosis, gene expression and stress granule formation in urothelial carcinoma cells. J Cell Mol Med 15: 1210-1224, 2011.

15. Wang XY, Penalva LO, Yuan H, Linnoila RI, Lu J, Okano H and Glazer RI: Musashi1 regulates breast tumor cell proliferation and is a prognostic indicator of poor survival. Mol Cancer 9: 221, 2010.

16. Liu X, Yang WT and Zheng PS: Msi1 promotes tumor growth and cell proliferation by targeting cell cycle checkpoint proteins p21, p27 and p53 in cervical carcinomas. Oncotarget 5: 10870-10885, 2014.

17. Götte M, Greve B, Kelsch R, Müller-Uthoff H, Weiss K, Kharabi Masouleh B, Sibrowski W, Kiesel L and Buchweitz O: The adult stem cell marker Musashi-1 modulates endometrial carcinoma cell cycle progression and apoptosis via Notch-1 and p21 WAF1/CIP1. Int J Cancer 129: 2042-2049, 2011.

18. Abdelmohsen K, Srikantan S, Kuwano Y and Gorospe M: miR-519 reduces cell proliferation by lowering RNA-binding protein HuR levels. Proc Natl Acad Sci USA 105: 20297-20302, 2008.

19. Busà R, Paronetto MP, Farini D, Pierantozzi E, Botti F, Angelini DF, Attisani F, Vespasiani G and Sette C: The RNA-binding protein Sam68 contributes to proliferation and survival of human prostate cancer cells. Oncogene 26: 4372-4382, 2007. 
20. Sutherland LC, Rintala-Maki ND, White RD and Morin CD: RNA binding motif (RBM) proteins: A novel family of apoptosis modulators? J Cell Biochem 94: 5-24, 2005.

21. Kaneko Y, Sakakibara S, Imai T, Suzuki A, Nakamura Y, Sawamoto K, Ogawa Y, Toyama Y, Miyata T and Okano H: Musashil: An evolutionally conserved marker for CNS progenitor cells including neural stem cells. Dev Neurosci 22: $139-153,2000$.

22. Okano H, Kawahara H, Toriya M, Nakao K, Shibata S and Imai T: Function of RNA-binding protein Musashi-1 in stem cells. Exp Cell Res 306: 349-356, 2005.

23. Rezza A and Plateroti M: M1621 Study of the RNA binding protein Musashil, An intestinal epithelial stem cell marker. Gastroenterology 136: A-396, 2009.

24. Vo DT, Subramaniam D, Remke M, Burton TL, Uren PJ, Gelfond JA, de Sousa Abreu R, Burns SC, Qiao M, Suresh U, et al: The RNA-binding protein Musashil affects medulloblastoma growth via a network of cancer-related genes and is an indicator of poor prognosis. Am J Pathol 181: 1762-1772, 2012.

25. Shi $\mathrm{C}$ and Zhang Z: miR-761 inhibits tumor progression by targeting MSI1 in ovarian carcinoma. Tumor Biol 37: 5437-5443, 2016.
26. Akasaka Y, Saikawa Y, Fujita K, Kubota T, Ishikawa Y, Fujimoto A, Ishii T, Okano $\mathrm{H}$ and Kitajima M: Expression of a candidate marker for progenitor cells, Musashi-1, in the proliferative regions of human antrum and its decreased expression in intestinal metaplasia. Histopathology 47: 348-356, 2005.

27. Wei JG, Zhao F and Sun AJ: Effect of Msil siRNA on proliferation of SW-480 cell line. Chin J Clin Exp Pathol, 2012.

28. Wang YF, Chen NS, Chung YP, Chang LH, Chiou YH and Chen CY: Sodium butyrate induces apoptosis and cell cycle arrest in primary effusion lymphoma cells independently of oxidative stress and p21 CIP1/WAF1 induction. Mol Cell Biochem 285: 51-59, 2006.

29. Møller MB: P27 in cell cycle control and cancer. Leuk Lymphoma 39: 19-27, 2000.

30. Liu DF, Ferguson K, Cooper GS, Grady WM and Willis J: p27 cell-cycle inhibitor is inversely correlated with lymph node metastases in right-sided colon cancer. J Clin Lab Anal 13: 291-295, 1999.

31. Coqueret O: New roles for $\mathrm{p} 21$ and p27 cell-cycle inhibitors: A function for each cell compartment? Trends in Cell Biol 13: 65-70, 2003. 